\title{
Replication study for the association of a single-nucleotide polymorphism, rs3746876, within $K C N J 15$, with susceptibility to type 2 diabetes in a Japanese population
}

\author{
Hisashi Fukuda ${ }^{1,2}$, Minako Imamura ${ }^{1}$, Yasushi Tanaka ${ }^{2}$, Minoru Iwata $^{3}$, Hiroshi Hirose ${ }^{4}$, Kohei Kaku ${ }^{5}$, \\ Hiroshi Maegawa ${ }^{6}$, Hirotaka Watada ${ }^{7,8}$, Kazuyuki Tobe ${ }^{3}$, Atsunori Kashiwagi ${ }^{6}$, Ryuzo Kawamori ${ }^{8}$ \\ and Shiro Maeda ${ }^{1}$
}

By an association mapping for the candidate locus in chromosome 21q, rs3746876 within KCNJ15 was shown to be associated with type 2 diabetes in Japanese populations. However, the association of rs 3746876 with type 2 diabetes has not been validated in an independent cohort. The aim of the present study was to ascertain the association of rs 3746876 with type 2 diabetes in an independent larger Japanese sample. We genotyped 7885 Japanese participants (4967 individuals with type 2 diabetes and 2918 control individuals) for rs 3746876 with polymerase-chain reaction-invader assay. The association of rs3746876 with type 2 diabetes was examined by using logistic regression analysis. Quantitative traits analyses for homeostasis model assessment (HOMA) of $\beta$-cell function, HOMA of insulin resistance, fasting plasma glucose, fasting immunoreactive insulin and body mass index (BMI) were performed in control individuals by using multiple-linear regression analysis. We observed a significant association of rs3746876-T with type 2 diabetes $(P=0.0281$, odds ratio $($ OR $)=0.82,95 \%$ confidence interval $(\mathrm{Cl}, 0.68-0.98)$ ), but the direction of effect was opposite to that in the original report. The association of rs3746876 with type 2 diabetes was more significant in obese patients $\left(\mathrm{BMI} \geqslant 25 \mathrm{~kg} \mathrm{~m}^{-2}, \boldsymbol{P}=0.0025,0 \mathrm{R}=0.62,95 \% \mathrm{Cl}\right.$, 0.45-0.84). We did not observe significant association of rs3746876 with any of the quantitative traits in the control individuals. We could not replicate the original finding for the association of rs 3746876 with type 2 diabetes, although rs3746876 was significantly associated with obese type 2 diabetes in the present Japanese population. Journal of Human Genetics (2013) 58, 490-493; doi:10.1038/jhg.2013.28; published online 18 April 2013

Keywords: case-control association study; Japanese; KCNJ15; single-nucleotide polymorphism; type 2 diabetes

\section{INTRODUCTION}

To date, over 60 susceptibility loci have been identified for type 2 diabetes, mostly through genome-wide association studies. ${ }^{1-20}$ Of these, several loci have been shown to be associated with type 2 diabetes in Japanese populations, ${ }^{21-26}$ whereas the remaining loci have not shown significant effects in ethnic populations other than those in the original reports. ${ }^{14,21-27}$ Although the power of these studies may be insufficient in some cases, genetic heterogeneity among different ethnicities may exist for some particular loci.

In 2010, by an association mapping in the previously identified candidate locus for type 2 diabetes, chromosome $21 \mathrm{q},{ }^{28}$ a single-nucleotide polymorphism, rs3746876, within the potassium inwardly-rectifying channel, subfamily J, member 15 gene (KCNJ15) was shown to be associated with type 2 diabetes in Japanese populations, but not in a Danish population. ${ }^{29}$ Subsequent biological analyses suggested that the KCNJ15 was involved in the type 2 diabetes susceptibility through an impairment of the insulin secretion from pancreatic $\beta$ cells. ${ }^{29,30}$ However, provided evidence for the association of rs 3746876 with type 2 diabetes was still not sufficient to include this locus in a list of confirmed type 2 diabetes locus, since the association of the SNP did not reach a level of genome-wide significance $\left(P<5 \times 10^{-8}\right)$, and there has been no report to replicate this association in an independent cohort.

${ }^{1}$ Laboratory for Endocrinology and Metabolism, RIKEN Center for Genomic Medicine, Yokohama, Japan; ${ }^{2}$ Division of Metabolism and Endocrinology, Department of Internal Medicine, St Marianna University School of Medicine, Kawasaki, Kanagawa, Japan; ${ }^{3}$ First Department of Internal Medicine, University of Toyama, Toyama, Japan; ${ }^{4}$ Health Center, Keio University School of Medicine, Tokyo, Japan; ${ }^{5}$ Division of Diabetes, Endocrinology and Metabolism, Department of Internal Medicine, Kawasaki Medical School, Kurashiki, Japan; ${ }^{6}$ Department of Medicine, Shiga University of Medical Science, Otsu, Japan; ${ }^{7}$ Department of Metabolism and Endocrinology, Juntendo University Graduate School of Medicine, Tokyo, Japan and ${ }^{8}$ Sportology Center, Juntendo University Graduate School of Medicine, Tokyo, Japan

Correspondence: Dr S Maeda, Laboratory for Endocrinology and Metabolism, RIKEN Center for Genomic Medicine, 1-7-22, Suehiro-cho, Turumi-ku, Yokohama, Kanagawa 230-0045, Japan. 
Table 1 Characteristics of participants

\begin{tabular}{|c|c|c|c|c|}
\hline & Case-control & Type 2 diabetes & Controls & P-value \\
\hline $\operatorname{Sex}(M: F)$ & $4697 / 2918$ & 2878:1819 & 1621:1297 & $<0.001^{a}$ \\
\hline BMI $\left(\mathrm{kg} \mathrm{m}^{-2}\right)^{\mathrm{d}}$ & $4697 / 2918$ & $23.9(16.5-32.7)$ & $22.6(17.2-29.7)$ & $<0.001^{c}$ \\
\hline HbAlc (NGSP, \%) & $4228 / 1327$ & $8.0 \pm 2.5$ & $5.4 \pm 0.3$ & $<0.001^{c}$ \\
\hline$P G\left(m m o l I^{-1}\right)^{d}$ & $2283 / 1325$ & $8.0(4.3-14.8)$ & $5.1(3.9-6.7)$ & $<0.001^{c}$ \\
\hline $\operatorname{HOMA}-\beta(\%)^{\mathrm{d}}$ & $-/ 893$ & & 52.5 (17.9-153.7) & \\
\hline
\end{tabular}

Abbreviations: BMI, body mass index; F-IRI, fasting imunoreactive insulin; HbAlc, glycated hemoglobin; HOMA-IR, homeostasis model assessment of insulin resistance; HOMA- $\beta$, HOMA of $\beta$ cell function; PG, plasma glucose.

aChi-square test.

bData are mean \pm s.d.

'Student's unpaired $t$-test.

${ }^{d}$ Geometric mean (95\% confidence interval).

In this study, we aim to evaluate the association of rs3746876 with susceptibility to type 2 diabetes in an independent Japanese population.

\section{SUBJECTS AND METHODS}

\section{Participants and DNA preparation and genotyping}

We enrolled 2664 type 2 diabetes patients who regularly visited the outpatient clinics of the Shiga University of Medical Science, Kawasaki Medical School, St Marianna University, Juntendo University and the University of Toyama. We additionally enrolled 2033 individuals who were registered as having type 2 diabetes in BioBank, Japan. ${ }^{7,14}$ We also examined 2918 controls, who underwent annual health check-ups at Keio University, St Marianna University or Toyama University Hospital, or from the general Japanese population registered in the Japanese SNP database. Individuals with fasting plasma glucose $(\mathrm{FPG}) \geqslant 7 \mathrm{mmol}^{-1}$, postprandial plasma glucose $\geqslant 11.1 \mathrm{mmol}^{-1}$ or glycated hemoglobin (HbAlc (NGSP)) $\geqslant 6.4 \%$ were excluded from the control group, if data were available. Fasting plasma insulin levels were obtained from 926 control individuals recruited at Keio University or Toyama University Hospital.

Diabetes mellitus was diagnosed according to the World Health Organization criteria, ${ }^{31}$ and type 2 diabetes was clinically defined by a gradual adult onset. We excluded patients who tested positive for antibodies to glutamic acid decarboxylase or who were diagnosed with mitochondrial disease or maturity-onset diabetes of the young. Obesity was defined as individuals with body mass index $(\mathrm{BMI}) \geqslant 25 \mathrm{~kg} \mathrm{~m}^{-2}$ according to the criteria of Japan Society for the Study of Obesity. ${ }^{32}$

\section{Ethics statements}

All participants agreed to the protocol of this study and provided written informed consent. The study protocol conformed to the provisions of the Declaration of Helsinki, and was approved by the ethics committees of RIKEN Yokohama Institutes and each of the participating institutes, that is, Shiga University of Medical Science, Kawasaki Medical School, St Marianna University, Juntendo University, the University of Toyama and Keio University.

\section{SNP genotyping}

Genotyping of rs3746876 was performed using the polymerase chain reaction invader assay. ${ }^{33}$ Genotyping success rate for the assay was $99.0 \%$ and concordance rate for this SNP in 126 randomly selected duplicated samples is $100 \%$.

\section{Statistical analysis}

We performed Hardy-Weinberg equilibrium tests according to the method described by Nielsen et al. ${ }^{34}$ To test the additive model for rs3746876 in $K C N J 15$, the difference in the genotype distribution of this SNP between cases and controls was evaluated by logistic regression analysis with or without adjusting for age, sex and BMI. Genotype was scored using an additive model for the analysis $(0,1$ and 2 for the homozygous of non-effect
Table2 Genotype distribution of rs3746876 in $K C N J 15$ in case and control groups

\begin{tabular}{lccc}
\hline & & Effect allele $(T)^{\mathrm{a}}$ & \\
& CC/CT/TT & frequency & P for HWE test \\
\hline Type 2 diabetes & $4291 / 346 / 8$ & 0.0390 & 0.7106 \\
Controls & $2628 / 254 / 10$ & 0.0476 & 0.1481 \\
\hline
\end{tabular}

Abbreviation: HWE, Hardy-Weinberg equilibrium.

aRisk allele reported in the previous report. ${ }^{29}$

allele, heterozygous and homozygous of effect allele, respectively). The association of this SNP with quantitative traits, BMI, FPG, fasting immunoreactive insulin, the homeostasis model assessment (HOMA) of $\beta$ cell function (HOMA- $\beta$ ) and the HOMA of insulin resistance (HOMA-IR) $)^{35,36}$ was evaluated using control individuals by multiple linear regression analysis in an additive association model. As the present Japanese samples have skewed distribution for BMI, FPG, F-IRI, HOMA-IR and HOMA- $\beta$, we performed the analyses for the quantitative traits using log-transformed BMI, FPG, F-IRI, HOMA-IR and HOMA- $\beta$.

Statistical analyses were performed using StatView software (SAS Institute, Cary, NC, USA). The level of significance was determined by a $P$ value of $<0.05$.

\section{RESULTS}

The characteristics of participants in the present study are presented in Table 1 . In type 2 diabetes cases, male rates, BMI and age are significantly higher than those in controls.

Genotype distribution of rs3746876 in KCNJ15 in the present study was in accordance with the Hardy-Weinberg equilibrium proportion (Table 2).

Results of an association study for rs 3746876 with type 2 diabetes in the present Japanese population are shown in Table 3. Rs3746876-T, which was reported to be associated with an increased risk of type 2 diabetes, ${ }^{29}$ was significantly associated with reduced risk of type 2 diabetes in the present Japanese population $(P=0.0281$, odds ratio $(\mathrm{OR})=0.82,95 \%$ confidence interval $(\mathrm{CI}, 0.68-0.98)$ ). Subsequent BMI-stratified analysis revealed that the association of rs3746876 with type 2 diabetes was significant only in obese subjects with type 2 diabetes $\left(\mathrm{BMI} \geqslant 25 \mathrm{~kg} \mathrm{~m}^{-2}, \quad P=0.0025, \quad \mathrm{OR}=0.62, \quad 95 \% \mathrm{CI}\right.$, $0.45-0.84$ ), but not in non-obese type 2 diabetes (BMI $<25 \mathrm{~kg} \mathrm{~m}^{-2}$, $P=0.2824, \mathrm{OR}=0.90,95 \% \mathrm{CI}, 0.73-1.10$, Table 3, Supplementary Table S1). The association of rs 3746876 with type 2 diabetes in a dominant or recessive association model was not stronger than that in an additive association model (Supplementary Table S2). 
Table3 Association of rs3746876 in KCNJ15 with type 2 diabetes in the Japanese population

\begin{tabular}{|c|c|c|c|c|c|c|}
\hline \multirow[b]{2}{*}{ Effect allele ${ }^{\mathrm{a}}$} & \multirow[b]{2}{*}{ Type 2 diabetes (n) } & \multirow[b]{2}{*}{ EAF (case-control) } & \multicolumn{2}{|c|}{ Unadjusted } & \multicolumn{2}{|c|}{ Adjusted $^{\mathrm{b}}$} \\
\hline & & & P-value & OR $(95 \% \mathrm{Cl})$ & P-value & OR $(95 \% \mathrm{Cl})$ \\
\hline $\mathrm{T}$ & All (4645) & $0.0390 / 0.0474$ & 0.0134 & $0.82(0.70-0.96)$ & 0.0281 & $0.82(0.68-0.98)$ \\
\hline $\mathrm{T}$ & Non-obese (2868) & $0.0415 / 0.0474$ & 0.1296 & $0.87(0.73-1.04)$ & 0.2824 & $0.90(0.73-1.10)$ \\
\hline $\mathrm{T}$ & Obese (1777) & $0.0349 / 0.0474$ & 0.0042 & $0.73(0.59-0.91)$ & 0.0025 & $0.62(0.45-0.84)$ \\
\hline
\end{tabular}

Abbreviations: $\mathrm{Cl}$, confidence interval; EAF, effect allele frequency; OR, odds ratio.

Results of logistic regression analysis are shown. Genotype was scored using an additive model for the analysis $(0,1$, and 2 for the homozygous of non-effect allele, heterozygous, and homozygous of effect allele, respectively).

aRisk allele reported in the previous report. ${ }^{29}$

${ }^{b}$ Adjusted for age, sex and log-transformed BMI.

Table 4 Association of rs3746876 in KCNJ15 with quantitative traits related to glucose metabolism in control individuals

\begin{tabular}{lccccc}
\hline Traits & HOMA-IR & HOMA- $\beta^{\mathrm{ab}}$ & FPG $^{\mathrm{ab}}$ & F-IR/ab & BM ${ }^{\mathrm{ac}}$ \\
\hline $\mathrm{n}$ & 893 & 893 & 1,325 & 926 & 2,918 \\
Effect $^{\mathrm{d}}$ & 0.001 & 0.027 & -0.001 & -0.029 & 0.004 \\
(SE) $_{P}$ & $(0.052)$ & $(0.055)$ & $(0.008)$ & $(0.049)$ & $(0.008)$ \\
$P$ & 0.9884 & 0.6201 & 0.9250 & 0.5521 & 0.5902 \\
\hline
\end{tabular}

Results of linear regression analysis. Genotype was scored using an additive model for the analysis $(0,1$, and 2 for the homozygous of non-effect allele, heterozygous, and homozygous of effect allele, respectively).

${ }^{a}$ Values are log-transformed for the analysis.

${ }^{\mathrm{b}}$ Adjusted for age, sex and log-transformed BMI.

${ }^{c}$ Adjusted for age and sex.

dEffect of previously reported risk allele (T).29

In quantitative traits analyses using control individuals, we did not observe any significant associations of rs3746876 with BMI, HOMA-IR, HOMA- $\beta$, FPG or F-IRI (Table 4 and Supplementary Tables S3-S5), although sample sizes might not be sufficiently large enough for the analyses (Supplementary Table S6).

\section{DISCUSSION}

In the present study, we performed a replication study for the association of rs3746876 within the KCNJ15, which was previously reported to be associated with non-obese Japanese type 2 diabetes, ${ }^{29}$ with susceptibility to type 2 diabetes in an independent Japanese population. We observed that rs3746876 was significantly associated with type 2 diabetes also in the present Japanese population, but effect direction was not consistent with that in the previous report.

In the original report by Okamoto et al., ${ }^{29}$ the T allele of rs3746876 within KCNJ15 was shown to be associated with increased risk of type 2 diabetes in 3268 Japanese individuals by an association mapping of SNPs in a candidate region of chromosome 21q. They also demonstrated that the association was more significant in non-obese type 2 diabetes $\left(\mathrm{BMI}<24 \mathrm{~kg} \mathrm{~m}^{-2}\right),{ }^{29}$ and $K C N J 15$ mRNA and protein were shown to be expressed in human pancreatic $\beta$ cells. ${ }^{29}$ Subsequently, it was shown that knockdown of kcnj15 by small interfering RNA resulted in increased insulin secretion in rat insulinoma cell lines, and in non-diabetic or diabetic Akita mice, ${ }^{30}$ indicating KCNJ15 plays a crucial role in the regulation of insulin secretion from pancreatic $\beta$ cells. KCNJ15 mRNA corresponding to rs3746876-T was shown to be degraded slower than that for rs3746876-C in cultured non-insulin-secreting cell lines, but the differences in the expression and/or function of KCNJ15 according to the genotype remains to be elucidated in pancreatic $\beta$ cells and in vivo.

In the present study, the reported risk allele $(\mathrm{T})$ showed a significant association with type 2 diabetes, but had a reverse direction of effect $(\mathrm{OR}<1.0)$. In addition, the association was significant only in obese type 2 diabetes $\left(\mathrm{BMI}>25 \mathrm{~kg} \mathrm{~m}^{-2}\right)$ in the present study, whereas the SNP was associated with non-obese type 2 diabetes in the original report. ${ }^{29}$ Sample size, 4697 cases and 2918 controls, in the present study was sufficiently large enough to replicate the original finding (estimated power is $100 \%$, Supplementary Table S7), and quality of the genotyping was well validated; therefore, these factors were not likely a principal cause for the discrepancy between the present study and the original study. Since we used current BMI, not maximum BMI, for the analysis, our results might be affected by therapeutic interventions, especially for type 2 diabetes cases. Comparing clinical characteristics between the present study and the original report, there was a significant difference in the allele frequency for control groups or in the sex ratio for type 2 diabetes cases (Supplementary Table S8); therefore, differences in environmental and/or regional factors between these studies might produce conflicting results, if the association of the KCNJ15 locus with type 2 diabetes truly exists. Since our sample collections have been made from the six distinct study groups for type 2 diabetes and from four distinct study groups for controls, we examined the differences in the genotype distributions for rs3746876 among six different collection groups for type 2 diabetes or among four collection groups for controls. The results indicated that genotype distributions for rs3746876 were not different among individual panels (Supplementary Table S9), suggesting that our present finding was not affected by sample heterogeneity.

Because control individuals in the present study were younger than type 2 diabetes patients, the control group might include several individuals who would develop the disease, and the possibility of type 2 error may be increased, although results were not affected by adjusting age. Then we evaluated the association of this SNP with type 2 diabetes using older control individuals (age $\geqslant 40, \geqslant 50$ or $\geqslant 60$, Supplementary Table S10). The results indicated that the rs3746876-T was consistently associated with less susceptibility to type 2 diabetes, and the association was strongest when we used control individuals with age $\geqslant 60$ for the analysis $(P=0.0039$, $\mathrm{OR}=0.71,95 \%$ CI, 0.56-0.89, Supplementary Table S10). Regarding the difference in the sex ratio, neither adjustment for sex nor stratification by sex affected the results of the association between rs3746876 and type 2 diabetes (Supplementary Tables S3 and S11). Since we did not perform a glucose tolerance test for selecting control individuals, our control group may include individuals with impaired glucose tolerance or subclinical diabetes. In addition, our type 2 diabetes group may include other types of diabetes. A possibility for type 2 error might be increased by including these inappropriate individuals; therefore, we did a quick lookup at association data for known type 2 diabetes loci in the present population to evaluate the feasibility of the present study. The results indicated that the associations of these known loci with Japanese type 2 diabetes were consistently observed in the present Japanese population 
(Supplementary Table S12), suggesting the present Japanese sample is feasible for the case-control association study.

Generally, the linkage disequilibrium pattern for particular loci may be different among different populations; therefore it is possible that rs3746876-C in the present population and rs3746876- $\mathrm{T}$ in the original population link to the same causal variant(s). However, the probability for this possibility is likely very low, because Japanese populations have been shown to be genetically homogeneous. $7,8,14,37$

Since the association was no longer significant when both results were combined by a meta-analysis $(P>0.05$ in both random-effect and fixed-effect models, data not shown), rs3746876 may not have a significant effect on susceptibility to type 2 diabetes, although further study is required for elucidating the contribution of this locus to conferring susceptibility to type 2 diabetes.

In conclusion, we performed a replication study using an independent larger Japanese population for the association of rs3746876 within KCNJ15, and observed a significant association of rs3746876 with type 2 diabetes. However, the present study failed to validate the original finding; therefore, it is considered that the KCNJ15 locus still does not have a place on the list of confirmed type 2 diabetes susceptibility loci at this moment.

\section{CONFLICT OF INTEREST}

The authors declare no conflict of interest.

\section{ACKNOWLEDGEMENTS}

We thank the technical staff of the Laboratory for Endocrinology and Metabolism at RIKEN Center for Genomic Medicine for their technical assistance. This work was partly supported by a Grant-in-Aid from the Ministry of Education, Culture, Sports, Science and Technology, Japan (to S.M.).

1 Sladek, R., Rocheleau, G., Rung, J., Dina, C., Shen, L., Serre, D. et al. A genome-wide association study identifies novel risk loci for type 2 diabetes. Nature 445, 881-885 (2007).

2 Zeggini, E., Weedon, M. N., Lindgren, C. M., Frayling, T. M., Elliott, K. S., Lango, H. et al. Replication of genome-wide association signal in UK samples reveals risk loci for type 2 diabetes. Science 316, 1336-1341 (2007).

3 Scott, L. J., Mohlke, K. L., Bonnycastle, L. L., Willer, C. J., Li, Y., Duren, W. L. et al. A genome-wide association study of type 2 diabetes in Finns detects multiple susceptibility variants. Science 316, 1341-1345 (2007).

4 Saxena, R., Voight, B. F., Lyssenko, V., Burtt, N. P., de Bakker, P. I., Chen, H. et al. Genome-wide association analysis identifies loci for type 2 diabetes and triglyceride levels. Science 316, 1331-1336 (2007).

5 Steinthorsdottir, V., Thorleifsson, G., Reynisdottir, I., Benediktsson, R., Jonsdottir, T., Walters, G. B. et al. A variant in CDKAL1 influences insulin response and risk of type 2 diabetes. Nat. Genet. 39, 770-775 (2007)

6 Zeggini, E., Scott, L. J., Saxena, R., Voight, B. F., Marchini, J. L., Hu, T. et al. Metaanalysis of genome-wide association data and large-scale replication identifies additional susceptibility loci for type 2 diabetes. Nat. Genet. 40, 638-645 (2008).

7 Unoki, H., Takahashi, A., Kawaguchi, T., Hara, K., Horikoshi, M., Andersen, G. et al. SNPs in KCNQ1 are associated with susceptibility to type 2 diabetes in East Asian and European populations. Nat. Genet. 40, 1098-1102 (2008).

8 Yasuda, K., Miyake, K., Horikawa, Y., Hara, K., Osawa, H., Furuta, H. et al. Variants in KCNQ1 are associated with susceptibility to type 2 diabetes mellitus. Nat. Genet. 40, 1092-1097 (2008).

9 Rung, J., Cauchi, S., Albrechtsen, A., Shen, L., Rocheleau, G., Cavalcanti-Proença, C. et al. Genetic variant near IRS1 is associated with type 2 diabetes, insulin resistance and hyperinsulinemia. Nat. Genet. 41, 1110-1115 (2009).

10 Lyssenko, V., Nagorny, C. L., Erdos, M. R., Wierup, N., Jonsson, A., Spégel, P. et al. Common variant in MTNR1B associated with increased risk of type 2 diabetes and impaired early insulin secretion. Nat. Genet. 41, 82-88 (2009).

11 Bouatia-Naji, N., Bonnefond, A., Cavalcanti-Proenca, C., Spars $\varnothing$, T., Holmkvist, J., Marchand, M. et al. A variant near MTNR1B is associated with increased fasting plasma glucose levels and type 2 diabetes risk. Nat. Genet. 41, 89-94 (2009).
12 Dupuis, J., Langenberg, C., Prokopenko, I., Saxena, R., Soranzo, N., Jackson, A. U. et al. New genetic loci implicated in fasting glucose homeostasis and their impact on type 2 diabetes risk. Nat. Genet. 42, 105-116 (2010)

13 Voight, B. F., Scott, L. J., Steinthorsdottir, V., Morris, A. P., Dina, C., Welch, R. P. et al. Twelve type 2 diabetes susceptibility loci identified through large-scale association analysis. Nat. Genet. 42, 579-589 (2010).

14 Yamauchi, T., Hara, K., Maeda, S., Yasuda, K., Takahashi, A., Horikoshi, M. et al. A genome-wide association study in the Japanese population identifies susceptibility loci for type 2 diabetes at UBE2E2 and C2CD4A-C2CD4B. Nat. Genet. 42, 864-868 (2010).

15 Shu, X. O., Long, J., Cai, Q., Qi, L., Xianq, Y. B., Cho, Y. S. et al. Identification of new genetic risk variants for type 2 diabetes. PLoS Genet. 6, e1001127 (2010).

16 Cho, Y. S., Chen, C. H., Hu, C., Long, J., Ong, R. T., Sim, X. et al. Meta-analysis of genome-wide association studies identifies eight new loci for type 2 diabetes in east Asians. Nat. Genet. 44, 67-72 (2012).

17 Kooner, J. S., Saleheen, D., Sim, X., Sehmi, J., Zhang, W., Frossard, P. et al. Genomewide association study in individuals of South Asian ancestry identifies six new type 2 diabetes susceptibility loci. Nat. Genet. 43, 984-989 (2011).

18 Imamura, M., Maeda, S., Yamauchi, T., Hara, K., Yasuda, K., Morizono, T. et al. A single-nucleotide polymorphism in ANK1 is associated with susceptibility to type 2 diabetes in Japanese populations. Hum. Mol. Genet. 21, 3042-3049 (2012).

19 Perry, J. R., Voight, B. F., Yengo, L., Amin, N., Dupuis, J., Ganser, M. et al. Stratifying type 2 diabetes cases by $\mathrm{BMI}$ identifies genetic risk variants in $\angle A M A 1$ and enrichment for risk variants in lean compared to obese cases. PLoS Genet. 8, e1002741 (2012).

20 Morris, A. P., Voight, B. F., Teslovich, T. M., Ferreira, T., Segrè, A. V., Steinthorsdottir, V. et al. Large-scale association analysis provides insights into the genetic architecture and pathophysiology of type 2 diabetes. Nat. Genet. 44, 981-990 (2012).

21 Omori, S., Tanaka, Y., Takahashi, A., Hirose, H., Kashiwagi, A., Kaku, K. et al. Association of CDKAL1, IGF2BP2, CDKN2A/B, HHEX, SLC3OA8, and KCNJ11 with susceptibility to type 2 diabetes in a Japanese population. Diabetes 57, 791-795 (2008).

22 Horikoshi, M., Hara, K., Ito, C., Nagai, R., Froguel, P. \& Kadowaki, T. A genetic variation of the transcription factor 7-like 2 gene is associated with the risk of type 2 diabetes in the Japanese population. Diabetologia 50, 747-751 (2007).

23 Hayashi, T., Iwamoto, Y., Kaku, K., Hirose, H. \& Maeda, S. Replication study for the association of TCF7L2 with susceptibility to type 2 diabetes in a Japanese population. Diabetologia 50, 980-984 (2007).

24 Onuma, H., Tabara, Y., Kawamoto, R., Shimizu, I, Kawamura, R., Takata, Y. et al. The GCKR rs780094 polymorphism is associated with susceptibility of type 2 diabetes, reduced fasting plasma glucose levels, increased triglycerides levels and lower HOMAIR in Japanese population. J. Hum. Genet. 55, 600-604 (2010).

25 Ohshige, T., Iwata, M., Omori, S., Tanaka, Y., Hirose, H., Kaku, K. et al. Association of new loci identified in European genome-wide association studies with susceptibility to type 2 diabetes in the Japanese. PLoS One 6, e26911 (2011).

26 Imamura, M., Iwata, M., Maegawa, H., Watada, H., Hirose, H., Tanaka, Y. et al. Genetic variants at $C D C 123 / C A M K 1 D$ and SPRY2 are associated with susceptibility to type 2 diabetes in Japanese population. Diabetologia 54, 3071-3077 (2011).

27 Imamura, M. \& Maeda, S. Genetics of type 2 diabetes: the GWAS era and future perspectives. Endocr. J. 58, 723-739 (2011).

28 Iwasaki, N., Cox, N. J., Wang, Y. Q., Schwarz, P. E., Bell, G. I., Honda, M. et al. Mapping gene influencing type 2 diabetes risk and BMI in Japanese subjects. Diabetes 52, 209-213 (2003).

29 Okamoto, K., Iwasaki, N., Nishimura, C., Doi, K., Noiri, E., Nakamura, S. et al. Identification of KCNJ15 as a susceptibility gene in Asian patients with type 2 diabetes mellitus. Am. J. Hum. Genet. 86, 54-64 (2010).

30 Okamoto, K., Iwasaki, N., Doi, K., Noiri, E., Iwamoto, Y., Uchigata, Y. et al. Inhibition of glucose-stimulated insulin secretion by $K C N J 15$, a newly identified susceptibility gene for type 2 diabetes. Diabetes 61, 1734-1741 (2012).

31 Alberti, K. G. \& Zimmet, P. Z. Definition, diagnosis and classification of diabetes mellitus and its complications. Part 1: diagnosis and classification of diabetes mellitus provisional report of a WHO consultation. Diabet. Med. 15, 539-553 (1998).

32 Examination Committee of Criteria for 'Obesity Disease' in Japan; Japan Society for the Study of Obesity. New criteria for 'obesity disease' in Japan. Circ. J. 66, 987-992 (2002).

33 Maeda, S., Tsukada, S., Kanazawa, A., Sekine, A., Tsunoda, T., Koya, D. et al. Genetic variations in the gene encoding TFAP $2 B$ are associated with type 2 diabetes mellitus. J. Hum. Genet. 50, 283-292 (2005).

34 Nielsen, D. M., Ehm, M. G. \& Weir, B. S. Detecting marker-disease association by testing for Hardy-Weinberg disequilibrium at a marker locus. Am. J. Hum. Genet. 63, 1531-1540 (1998)

35 Matthews, D. R., Hosker, J. P., Rudenski, A. S., Naylor, B. A., Treacher, D. F. \& Turner, R. C. Homeostasis model assessment: insulin resistance and beta-cell function from fasting plasma glucose and insulin concentrations in man. Diabetologia 28, 412-419 (1985).

36 Wallace, T. M., Levy, J. C. \& Matthews, D. R. Use and abuse of HOMA modeling. Diabetes Care 27, 1487-1495 (2004)

37 Yamaguchi-Kabata, Y., Nakazono, K., Takahashi, A., Saito, S., Hosono, N., Kubo, M. et al. Japanese population structure, based on SNP genotypes from 7003 individuals compared to other ethnic groups: effects on population-based association studies. Am. J. Hum. Genet. 83, 445-456 (2008). 